Bio - grafía. Escritos sobre la Biología y su Enseñanza. ISSN 2027-1034

Edición Extraordinaria. p.p. 865 - 873

Memorias del IX Encuentro Nacional de Experiencias en Enseñanza de la Biología y la Educación Ambiental. IV Congreso Nacional de Investigación en Enseñanza de la Biología.

\title{
ENSEÑANZA-APRENDIZAJE DE LA OFIDIOFAUNA EN LA REGIÓN SUR DE COLOMBIA: UN PROBLEMADE INVESTIGACIÓN Y UNA REVISIÓN DE ANTECEDENTES
}

\section{TEACHING-LEARNING OF OPHIDIOFAUNA IN THE SOUTHERN REGION OF COLOMBIA: A RESEARCH PROBLEM AND A BACKGROUND CHECK}

\author{
Gómez Cubillos, Dana Lizeth ${ }^{1}$ \\ Herrera Polania, Juan Felipe ${ }^{1}$ \\ Mosquera, Jonathan Andrés ${ }^{2}$ \\ Amórtegui Cedeño, Elías Francisco ${ }^{3}$ \\ Gaitán López, Erik Camilo ${ }^{4}$
}

\section{RESUMEN}

Presentamos una propuesta de investigación en torno a la enseñanza-aprendizaje sobre la conservación de la ofidiofauna en el contexto del municipio de Rivera, Huila. Este estudio se enmarca en una metodología con enfoque mixto, en el cual, se va a aplicar un cuestionario como fuente de recolección de información que tiene como objetivo indagar las concepciones del estudiantado. Se espera establecer en un segundo momento una escala Likert para medir algunas representaciones sociales sobre conservación, para ello se empleará material didáctico, como guías de campo, con el fin de abordar procesos de enseñanza en campo. Los métodos a utilizar en esta investigación serán el análisis de contenido y el análisis básico estadístico correlacional, de manera apropiada para cada instrumento. La población de estudio a intervenir en esta investigación corresponde a estudiantes de Noveno grado de la Institución Educativa Núcleo Escolar el Guadual. Para el caso de esta ponencia, se plasman resultados parciales en relación al estado del arte de la temática de investigación, la construcción del instrumento principal diseñado y validado, así como algunas observaciones realizadas en visitas preliminares con la comunidad.

PALABRAS CLAVE: Enseñanza, Aprendizaje, Concepciones, Ofidiofauna.

\footnotetext{
${ }^{1}$ Estudiantes del Programa de Licenciatura en Ciencias Naturales: Física, Química y Biología. Universidad Surcolombiana. felipepolania19@gmail.com dana.9625@hotmail.com

2 Docente de Cátedra e Investigador, Licenciatura en Ciencias Naturales: Física, Química y Biología, Universidad Surcolombiana. jonathan.mosquera@usco.edu.co

3 Docente de Planta Tiempo Completo, Licenciatura en Ciencias Naturales: Física, Química y Biología, Universidad Surcolombiana. elias.amortegui@usco.edu.co

${ }^{4}$ Licenciado en Ciencias Naturales y Educación Ambiental. Secretaría de Ambiente, Alcaldía de Neiva.camilo179201@hotmail.com
} 
Bio - grafía. Escritos sobre la Biología y su Enseñanza. ISSN 2027-1034

\title{
Edición Extraordinaria. p.p. 865 - 873
}

\author{
Memorias del IX Encuentro Nacional de Experiencias en Enseñanza de la Biología y la \\ Educación Ambiental. IV Congreso Nacional de Investigación en Enseñanza de la \\ Biología.
}

\begin{abstract}
We present a research proposal on teaching-learning about the conservation of Ofidiofauna in the context of the municipality of Rivera, Huila. This study is part of a methodology with a mixed approach, in which a questionnaire is to be applied as a source of information collection aimed at investigating the conceptions of students. It is expected to establish a Likert scale at a second time to measure some social representations on conservation, for it will be used didactic material, as field guides, in order to address processes of teaching in the field. The methods to be used in this research will be content analysis and correlational statistical basic analysis, in an appropriate manner for each instrument. The study population to intervene in this research corresponds to ninth-grade students of the school core educational institution El Guadual. In the case of this paper, partial results are reflected in relation to the state of the Art of the research theme, the construction of the main instrument designed and validated, as well as some observations made in preliminary visits with the community.
\end{abstract}

KEY WORDS: Teaching, learning, conceptions, Ophidiofauna.

\section{INTRODUCCIÓN}

En Colombia según el Serpentario Nacional existen alrededor de 320 especies en el país, que habitan desde el nivel del mar hasta unos $3500 \mathrm{msnm}$ y tan solo el $8 \%$ son venenosas. Estas especies representan el 9\% a nivel mundial, que se distribuyen en 10 familias (Anilidae, Anomalepidae, Boidae, Colubridae, Elapidae, Dipsadidae, Leptotyphlopidae, Tropidophidae, Typhlopidae, y Viperidae), situación que le confiere a Colombia, ser uno de los cinco países más ricos en serpientes (Uetz y Hosek 2014).

La mayor parte de las especies de serpientes en Colombia son inofensivas o poseen toxinas de baja potencialidad, las cuales no ponen en riesgo la vida humana. Algunos ofidios desarrollan dentición opistoglifa, la cual tienen un bajo perfil tóxico, de igual forma existen especies aglifas que carecen de colmillos y por consiguiente no son venenosas (Johanbocke, 1974 y Minton, 1979), las especies que presentan dentición proteroglifa o solenoglifa son las que presentan mayor riesgo para los humanos, como la familia de los elápidos (corales verdaderas) y vipéridos (talla equis, cascabeles). Las especies de estas dos familias son las causantes principales de los accidentes ofídicos en Colombia, representando así un problema de salud pública, que a su vez toman gran importancia clínica debida a su amplia variación ontogenética y geográfica que explica la baja efectividad de algunos sueros antiofídicos (Mendoza, 2015).

Por otra parte, la mayoría de los colombianos tiene una reacción negativa al encontrarse con una serpiente, demostrando así un desagrado u odio. Este odio proviene del desconocimiento de las personas, pues la mayoría asumen que todas las serpientes son venenosas. Esta tendencia, surge de diferentes mitos populares, en el cual, estigmatizan en alto grado a este tipo de organismos. (Lynch, 2012). 


\title{
Bio - grafía. Escritos sobre la Biología y su Enseñanza. ISSN 2027-1034
}

\section{Edición Extraordinaria. p.p. $865-873$}

\author{
Memorias del IX Encuentro Nacional de Experiencias en Enseñanza de la Biología y la \\ Educación Ambiental. IV Congreso Nacional de Investigación en Enseñanza de la \\ Biología.
}

De la misma manera, dentro de los ecosistemas las serpientes tienen un rol ecológico característico, ya que al ser depredadoras controlan algunas plagas como roedores y regulando la sobrepoblación de su misma especie, manteniendo el equilibrio ecológico en zonas donde ellas habitan (Lynch, 2012), además cumplen un papel importante en la salud pública puesto que son necesarias para la elaboración y fabricación de suero antiofídico para tratar mordeduras accidentales (Pough et al., 1998).

En relación con el programa de Licenciatura en Ciencias Naturales: Física, Química y Biología, de la Universidad Surcolombiana, es de vital importancia que sus estudiantes y egresados tengan una formación integral, con espíritu investigativo y capacidad de generar nuevos espacios formativos. Por tal, motivo este proyecto tiene como objetivo central contribuir desde el ámbito investigativo a la enseñanza y aprendizaje sobre conservación de la ofidiofauna.

\section{METODOLOGÍA}

En cuanto a la metodología de investigación, el estudio es de enfoque tipo mixto. Así mismo la investigación mixta es un proceso integral en el cual se incluyen técnicas, métodos, procesos y diferentes herramientas e instrumentos que provienen del enfoque cualitativo como también del enfoque cuantitativo. Además este enfoque de investigación es mucho más complejo, ya que permite ver desde diferentes matices como lo son percepciones, concepciones a partir de la observación, la convivencia y la interacción con nuestra población objeto de estudio. Hernández et al. (2006),

Basándonos en lo dicho anteriormente se propone el análisis de contenido como método de estudio que según Piñuel (2002), es una técnica utilizada comúnmente en la investigación cualitativa, lo denomina también como el conjunto de procedimientos interpretativos de productos comunicativos que proceden de procesos singulares de comunicación previamente registrados tiene como objetivo la elaboración y el procesamiento de datos relevantes sobre las condiciones mismas en que se han producido aquellos textos, en el cual se pretende elaborar diferentes categorías y generar una red temática entorno al problema de investigación en este caso la enseñanzaaprendizaje sobre la conservación de la ofidiofauna.

Por otra parte, se aplicará el método análisis correlacional estadístico, el cual permite hacer análisis estadísticos que básicamente serían: informes, comparación de medias, correlaciones para cualquier nivel de medición de variables, análisis de varianza vectorial en varias direcciones, reducciones de datos, escalas, validación compleja, series de tiempo, modelo lineal general, regresión, entre otros., lo cual se hará mediante el programa SPSS (Hernández, et al., 2006).

Para desarrollar este marco metodológico y poder alcanzar los objetivos de la investigación se plantea una serie de instrumentos: 
Bio - grafía. Escritos sobre la Biología y su Enseñanza. ISSN 2027-1034

Edición Extraordinaria. p.p. 865 - 873

Memorias del IX Encuentro Nacional de Experiencias en Enseñanza de la Biología y la

Educación Ambiental. IV Congreso Nacional de Investigación en Enseñanza de la Biología.

- Cuestionario. Además de las preguntas abiertas, se utilizó una escala de apreciaciones tipo escala Likert donde el estudiante tuvo la posibilidad de generar un punto de vista en torno a una serie de secuencias, patrones e ideas para generar una conducta particular.

- Observación participante. Esta técnica de recolección de información se implementará en el marco de la práctica pedagógica I, el cual permitirá un contacto directo con la población de estudio, pues al ser practicantes docentes en ejercicio y al tiempo investigadores, haremos reflexión en torno al aula, analizando diferentes procesos como lo es, la convivencia, comportamiento, disposición que tienen los estudiantes para las temática de la intervención y para el aprendizaje de las ciencias naturales.

- Intervención didáctica. Esta sesión durará ocho semanas, en donde se diseñarán dos salidas de campo, se hará trabajos prácticos y su temática se basará en la evolución, mitos, morfología, fisiología, alimentación, reproducción, ecología y conservación. Así mismo, se tendrán en cuenta las concepciones recabadas en el cuestionario inicial, para así mismo plantear estrategias que permitan el fortalecimiento de las concepciones para que haya una movilización de esas concepciones alternas hacia unas concepciones favorables de acuerdo a la didáctica de las ciencias naturales.

\section{RESULTADOS Y DISCUSIÓN}

A continuación, se presentan los principales hallazgos de la revisión de antecedentes sobre la enseñanza y el aprendizaje de la Ofidiofauna, considerando experiencias a nivel rural y urbano, y valorando la importancia de las salidas de campo. La revisión documental se realizó a nivel Internacional, nacional y regional, en bases de datos de acceso libre tales como Redalyc, Scielo, Scopus, SienceDirect y algunas revistas de alto impacto de habla hispana como Eureka, Bio-grafía y Enseñanza de las Ciencias.

En el plano internacional, las investigaciones se han centrado en el análisis de concepciones y conductas que presentan algunos estudiantes y profesores de educación escolar entorno a diferentes especies animales domésticos y animales no carismáticos, como caso específico de este estudio, las serpientes. Los estudios de Ron y Amy (2015) e Iztok Tomažič (2011), permiten visualizar los imaginarios de las poblaciones de estudio hacia especies de estos organismos, que por lo general son de desagrado y repudio, generando actitudes negativas y desinterés sobre aprender y enseñar acerca de las serpientes. Así mismo trabajos como el de Prokop, Özel y Uşak (2009), examinan variaciones de actitudes humanas hacia los ofidios en diferente contexto culturales de países de medio oriente como Turquía y Eslovaquia, en el cual se identifican diferentes dimensiones negativas, las influencias de los mitos populares y el interés por la biología de las serpientes en estudiantes universitarios. 


\title{
Bio - grafía. Escritos sobre la Biología y su Enseñanza. ISSN 2027-1034
}

\section{Edición Extraordinaria. p.p. $865-873$}

\author{
Memorias del IX Encuentro Nacional de Experiencias en Enseñanza de la Biología y la \\ Educación Ambiental. IV Congreso Nacional de Investigación en Enseñanza de la \\ Biología.
}

En el plano nacional es pertinente mencionar a Lynch (2012), quien con su estudio deduce que la mayoría de colombianos sienten odio o miedo hacia las serpientes teniendo en cuenta que esto deriva del desconocimiento lo que nos hace pensar que una de las soluciones va desde la parte educativa y el hecho de concientizar a la población colombiana sobre esta problemática. Por otra parte, Velandia (2015), ha hecho un gran aporte a la construcción de una sociedad colombiana comprometida con la conservación de la fauna silvestre y la flora de nuestro país generando estrategias educativas enfatizadas a la conservación con su estudio, pues, mediante la elaboración y aplicación de las actividades de la guía, el cual buscó generar procesos de sensibilización permitieron desarrollar procesos de reflexión crítica, partiendo desde las concepciones que poseen los miembros de las instituciones educativas y pobladores de la región, en torno a la conservación de la biodiversidad.

También es importante mencionar algunos autores, tales como Dávila y Buelvas (2009), Vargas et al.,(2011), Cortés y Toledo (2013), Ruíz (2014) y Latorre (2016); que han desarrollado una serie de investigaciones centradas principalmente en el registro e identificación de la diversidad de las especies de serpientes que se encuentran en algunos departamentos de la región caribe, la estimación de mortalidad de estos organismos por atropellamiento y la compresión del sentido de respeto a la vida de la ofidiofauna, lo que impulsa a generar estrategias que disminuyan estos índices y se promueva su conservación. A pesar de que se han realizado las investigaciones anteriormente nombradas se ve la gran necesidad de realizar proyectos con un enfoque hacia la educación donde las personas conozcan sobre las serpientes, los beneficios que representan en los ecosistemas.

En el departamento de Huila se han realizado algunos estudios como el Castrillón et al. (2015) y Moreno y Quintero (2015), que se han focalizado en caracterizaciones disciplinares biológicas de algunos organismos como odonatos y reptiles, dejando a un lado el proceso de formación en una cultura ambiental sustentable e incentivando a la conservación de las especies objetos de estudios en las investigaciones. Por otra parte, se registran pocos estudios con enfoque didáctico, entre esos están los estudios de Valenzuela et al. (2015), Flórez y Gaitán (2015) y Rivera, (2016), los cuales permiten evidenciar la manera en como favorecen la enseñanza-aprendizaje de la conservación de distintos grupos faunísticos como aves, quirópteros y arácnidos con estudiantes de instituciones educativas públicas en algunas zonas rurales y urbanas de la región.

A pesar de estas investigaciones, es evidente que en el contexto regional no existen trabajos investigativos y educativos sobre la enseñanza de la conservación de ofidiofauna. Siendo necesario que se generen este tipo de estudios por el alto desconocimiento de este grupo faunístico en la región local, partiendo desde centros educativos, incentivando y concientizando a niños, jóvenes y adultos al cuidado de las serpientes y al quehacer frente a accidentes ofídicos en nuestro departamento mediante estrategias didácticas en la cual se generen cambios actitudinales de la población opita. 
Bio - grafía. Escritos sobre la Biología y su Enseñanza. ISSN 2027-1034

Edición Extraordinaria. p.p. 865 - 873

Memorias del IX Encuentro Nacional de Experiencias en Enseñanza de la Biología y la Educación Ambiental. IV Congreso Nacional de Investigación en Enseñanza de la Biología.

\section{Construcción del Cuestionario}

Esta herramienta nos permite adquirir conocimiento acerca de las ideas previas con el fin de conocer, analizar, caracterizar el conocimiento y las concepciones acerca de los ofidios y su conservación. El cuestionario, consta de once (11) preguntas abiertas donde se tienen en cuenta ocho (8) temáticas. Estas preguntas posibilitan que los estudiantes tengan respuestas libres y que su conocimiento esté plasmado con sus propias palabras desapareciendo así la posibilidad que se limite.

Las temáticas son las siguientes:

- Mitos populares: en donde se cuestiona sobre alguna historia o mito que haya contado sus padres y abuelos o escuchado en la comunidad donde vive.

- Morfología: se pregunta si alguna vez ha visto una serpiente y el conocimiento que tienen sobre estas, tendrán que dibujarla e identificar sus partes.

- Fisiología: mediante el planteamiento de una supuesta noticia ("serpiente venenosa mordió a campesino cuando cogía café") se le pregunta al estudiante si cree que todas las serpientes son venenosas, donde tendrán que justificar la respuesta; también se les cuestiona la razón del por qué las serpientes sacan su lengua constantemente.

- Alimentación: indagando de qué creen que se alimentan y si algún otro organismo puede alimentarse de las serpientes.

- Reproducción: interrogar sobre la manera en que se reproducen las serpientes.

- Ecología: se cuestiona acerca de la importancia biológica en los ecosistemas.

- Conservación: se les plantea la situación en la cual se supone que el estudiante es un investigador de serpientes Huilense y se ha dado cuenta que en la vereda el Pedregal en Rivera, los campesinos afirman que han visto una nueva serpiente por los pastizales, se le indaga cómo haría para atraparla y estudiarla de tal modo que beneficie a la conservación de este grupo faunístico.

- Evolución: se indaga acerca del parentesco de los dinosaurios con las serpientes y el por qué. 
Bio - grafía. Escritos sobre la Biología y su Enseñanza. ISSN 2027-1034

Edición Extraordinaria. p.p. 865 - 873

Memorias del IX Encuentro Nacional de Experiencias en Enseñanza de la Biología y la

Educación Ambiental. IV Congreso Nacional de Investigación en Enseñanza de la Biología.

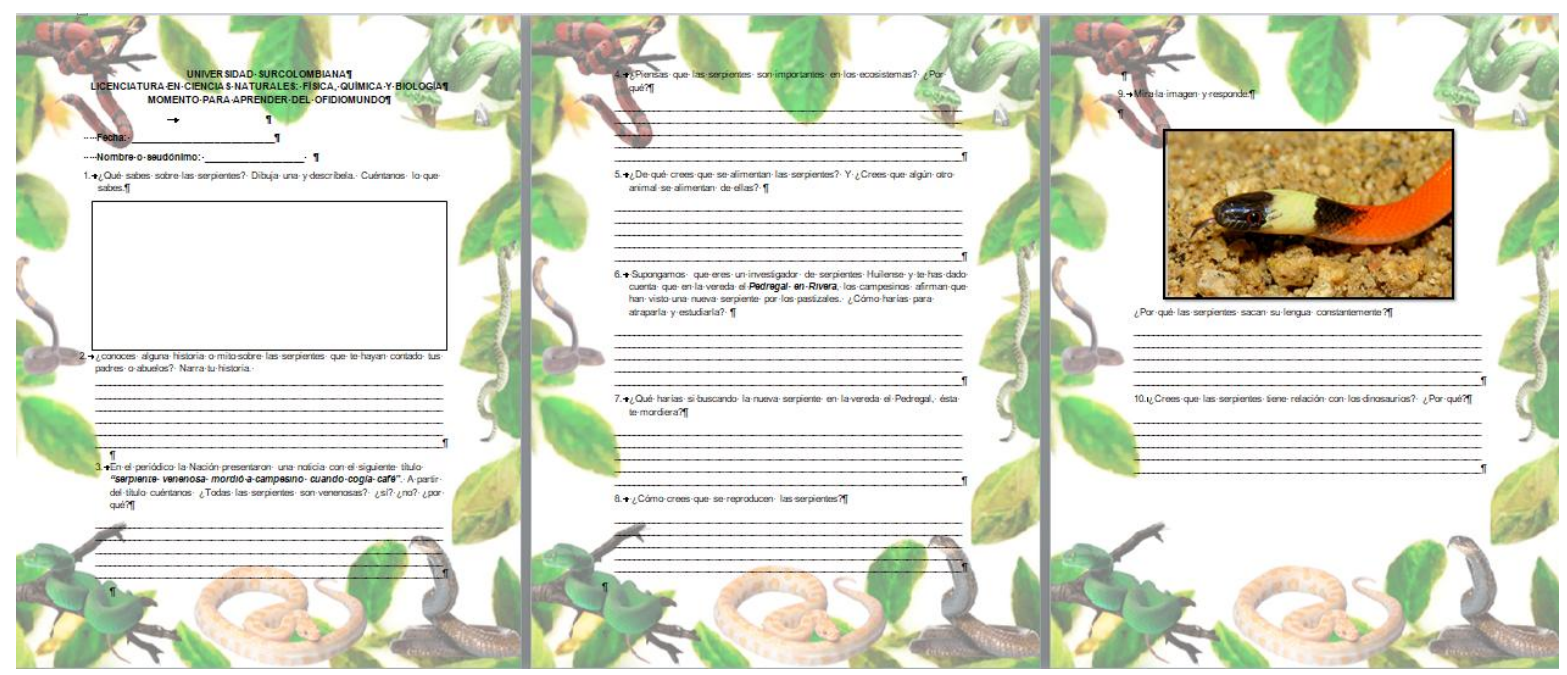

Posteriormente el cuestionario ha sido validado por tres expertos en el tema de la investigación, un doctor en ciencias experimentales docente de planta de la Universidad de Valencia, un licenciado de la Universidad Surcolombiana y candidato a magister de la Universidad del Tolima y finalmente un magister en educación de la Universidad Pedagógica Nacional. En la validación de este cuestionario se tienen en cuenta varios elementos tales como las características de la pregunta respecto a su potencia para indagar ideas previas, la claridad de la proposición, el lenguaje utilizado y la redacción empleada.

\section{CONCLUSIONES}

El campo de estudio de la enseñanza y aprendizaje de la ofidiofauna en el departamento del Huila ha sido poco explorado, debido a que se la mayoría de estudios se han centrado en términos de diversidad, es decir, en el ámbito disciplinar de la biología. En el caso particular de este grupo faunístico, hay ausencia de estudios indagatorios sobre las concepciones y estrategias de conservación que desarrollen procesos de formación y generen una cultura ambiental en la población opita.

En el municipio de Rivera, es escaso el conocimiento que se tiene entorno a procesos de enseñanza y aprendizaje en el ámbito de la biología, por lo tanto las estrategias que se utilizan se enmarcan para proyectos productivos, en el cual, se desconocen diferentes iniciativas didáctico-pedagógicas para el desarrollo de procesos de educación ambiental y conservación de diferentes especies de fauna presentes en el municipio. 
Bio - grafía. Escritos sobre la Biología y su Enseñanza. ISSN 2027-1034

Edición Extraordinaria. p.p. 865 - 873

Memorias del IX Encuentro Nacional de Experiencias en Enseñanza de la Biología y la Educación Ambiental. IV Congreso Nacional de Investigación en Enseñanza de la Biología.

\section{BIBLIOGRAFÍA}

Castrillón, G., Salazar, S. A., Amórtegui, E. F. y Palacino F (2015). Diversidad de odonatos en el centro de investigación y educación ambiental "la tribuna", NeivaHuila. Neiva, Huila: Universidad Surcolombiana.

Cortes-Ávila, L., y Toledo, J. J. (2013). Estudio de la diversidad de serpientes en areas de bosque pertubado y pastizal en San Vicente del Caguan (Caquetá), Colombia. Actual Biol, 185-197 pp.

Davila S, J., y Buelvas S, J. (2009). Reporte de algunas especies de ofidios presentes en el departamento de Sucre, Colombia. Revista Colombiana de Ciencia Animal, 273$278 \mathrm{pp}$.

Flórez, J. M. y Gaitán, E. C. (2015). Enseñanza de la avifauna a través de salidas de campo en estudiantes de cuarto y quito de primaria de la Institución Educativa Guacirco sede Peñas Blancas, Vereda Peñas Blancas. Neiva, Huila: Universidad Surcolombiana.

Hernandez, S., Ferandez, C. y Baptista, P. (2006). Metodología de la Investigación. Mexico, D.F.: McGraw-Hill/Interamericana Editores.

Johanbocke, M. (1974). Effects of a bite from Conophis lineatus (Squamata: Colubridae). . Bulletin, Philadelphia Herpetological Society, 22:39.

Lynch, J. D. (2012). El Contexto de las Serpientes e Colombia con un Análisis de las Amenazas en Contra de su Conservación, (1). Revista Académica Colombiana de Ciencias, 435-449 pp.

Mendoza, J. (2015). El reto de la conservación de serpientes en Colombia. . Apuntes Científicos uniandinos. , 36-47 PP.

Minton Jr, S. A. (1979). Beware: nonpoisonous snakes. . Clin. Toxicol, 15, 259-265 pp.

Moreno, R. y Quintero, S. (2015). Reptiles del Valle seco del Río Magdalena (Huila, Colombia). Caldasia, Vol. 33, No. 1.

Piñuel, J. L. (2002). Epistemología, metodología y técnicas de análisis de contenido. . Estudios de sociolinguística, 1-42 pp.

Pough, F. H., Andrews, R. M., Cadle, J. E., Crump, M. L., Savitzky, A. H., y Wells, K. D. (1998). Herpetology. New Jersey: Prentice-Hall.

Prokop, P., Özel, M. y Uşak, M. (2009). Cross-cultural comparison of Student Attitdes toward Snakes. . Society \& Animals, 224-240.

Rivera, S. (2016). Enseñanza y aprendizaje de la quirópterofauna a través del diseño y aplicación de una unidad didáctica dirigida a estudiantes de octavo grado de la 
Bio - grafía. Escritos sobre la Biología y su Enseñanza. ISSN 2027-1034

Edición Extraordinaria. p.p. 865 - 873

Memorias del IX Encuentro Nacional de Experiencias en Enseñanza de la Biología y la

Educación Ambiental. IV Congreso Nacional de Investigación en Enseñanza de la Biología.

Institución Educativa Técnico Superiór de Neiva. Neiva, Huila: Universidad Surcolombiana.

Ruiz, P. (2014). Ofidios del corregimiento de San Rafael del Pirú, Valencia (Córdoba, Colombia). Rev. Colombiana Cienc. Anim. .

Tomažič, L. (2011). Pre-Servicio de Biología de los Maestros' y la Escuela Primaria' Actitudes de los alumnos hacia y Conocimiento sobre las Serpientes. . Eurasia Journal of Mathematics, Science \& Technology Education, 7(3), , 161-171.

Uetz, P. y J. Hosek. (2014). The reptileDatabase. . Obtenido de The reptileDatabase. : http://www.reptile-database.org.

Valenzuela, J., González, J., Lacava, M., García, F. y Amórtegui, E.F. (2015). Arácnidos del PNN cueva de los guácharos. . Neiva, Huila: Editorial Universidad Surcolombiana.

Vargas-Salinas, F. (2011). Mortalidad por Atropello Vehicular y Distribución de Anfibios y Reptiles en un Bosque Subandino en el Occidente de Colombia. Caldasia, 33(1), 121-138.

Velandia, W. (2015). La enseñanza de la ecología de la herpetofauna en la conservación de los humedales de la Orinoquía Colombiana en Puerto Carreño, Vichada. Biografía Escritos en la Biología y su enseñanza. Vol. 8 No 14.

Wagler, R. y Wagler, A. (2015). Assessing the Attitudes and Beliets of Preservice Middle School Science Teachers toward Biologically Diverse Animals. . Iser, International J. Sci. Env. Ed., 10. 